\title{
Réalisation de réseaux sur polymères par laser UV
}

\author{
M.C. Castex, A. Fischer, D. Simeonov, D. Adès ${ }^{1}$ et A. Siove ${ }^{1}$ \\ Laboratoire de Physique des Lasers, CNRS, Université Paris-Nord, France \\ ${ }^{1}$ Laboratoire de Recherches sur les Macromolécules, Université Paris-Nord, Villetaneuse, \\ France
}

\begin{abstract}
Résamé : Nous présentons ici une méthode très simple de réalisation de réseaux d'amplitude de pas sub-micronique sur des firms polymères à base de carbazole déposés sur des substrat de silice. Les réseaux sont fabriqués an irradiant avec un faiscean laser à $193 \mathrm{~mm}$ un masque de phase placé au contact du film. La qualité des réseaux obtenus permet désormais d'envisager la réalisation de micro-savités lasers de type planaire permettant de sélectionner me émission laser monomode autour de $400 \mathrm{~nm}$
\end{abstract}

\section{INTRODUCTION}

La réalisation de diodes electroluminescentes organiques fait lobjet dune intense recherche internationale tant au plan academique qu'au plan industriel. La fabrication de tels dispositifs présente en effet un enjeu économique considérable touchant en particulier le secteur de l'aftichage électronique à bas coût et sur de grandes surfaces. De premiers produits industriels (écrans de téléphones mobiles, auto-radio,...) ont fait leur apparition en 1997 avec Pioneer suivi, entre autres, par Philips, Cambridge Display Technology, Seiko, Epson [1]. Plus en amont, le développement de micro-lasers plastiques fait également l'objet d'une recherche active [2-3]. Mais, qu'il s'agisse d'écrans plats ou de micro-lasers, l'optimisation des propriétés d'émission du materiau organique constitue une priorité.

De nombreuses solutions ont été trouvées pour contrôler les propriétés émissives (intensité, couleur, durée de vie, stabilité...) des matériaux organiques électroluminescents. Ainsi, différents types d'émetteurs rouges et verts, intenses et stables, ont pu être créés [4-5] par ingénierie moléculaire, permettant de réaliser des dispositifs performants (diodes électroluminescentes, micro-lasers pompés optiquement). Dans le visible, le premier laser à gain distribué (DFB, Distibnted Feedbach), basé sur l'insertion d'un reseau de Bragg dans le milieu polymère actif, a fait son apparition des 1970 avec les expériences de H. Kogelnik et C.V. Shank [6]. Par contre, la synthèse et l'utilisation d'émetteurs bleus est toujours d'actualité [7] et la fabrication d'un laser plastique bleu autour de $400 \mathrm{~nm}$ se heurte encore aux difficultés de fabrication of'un micro-résonateur. En effet, les conditions de rétroaction DFB nécessite la réalisation sur/dans le film polymère, d'un réseau de pas sub-micronique $(\Lambda \approx 130 \mathrm{~nm})$ pour satisfaire à la condition de Bragg ( $m \lambda_{\text {Fim }}=2 n_{\text {eff }} A$ ) dans l'orare $m=1$, avec un indice effectif $n_{\text {eff }}$ associé au mode de propagation.

Nous présentons ici une méthode simple de réalisation de réseaux d'amplitude de pas submicronique sur des films polymères à base de carbazole déposés sur des substrat de silice. Les réseaux sont fabriqués en irradiant avec un faisceau laser à $193 \mathrm{~nm}$ un masque de phase placé au contact du film. La qualité des réseaux obtenus permet désormais d'envisager la réalisation de micro-cavités lasers de type planaire permettant de selectionner une émission laser monomode autour de $400 \mathrm{~nm}$.

\section{MATERUAUX POLYMERES}

Depuis 1998, nous nous interessons aux proprietés de lummescence des polycarbazoles et de leurs précuseurs moleculaites. Ces polymères, synthétisés an LRM, pemettent c'obtenir une énission bleue caractéristique de la dyade bicarbazyle ou, quand ils sont modinés par des groupements spécifiques, des emissions allant du vert au rouge [8.9]. Les polymès sont thernostables (leur decomposition themique 
n'étant appréciable qu'au-dessus de $425^{\circ} \mathrm{C}$ ) et ils sont solubles dans les solvants organiques usuels. Ils présentent donc l'avantage de pouvoir être facilement mis sous forme de films par spin-coating sur divers substrats. Les composés moléculaires peuvent être obtenus à l'état de couche mince par évaporation thermique ou être utilisés comme dopants dans une matrices polymère telle que le PMMA.
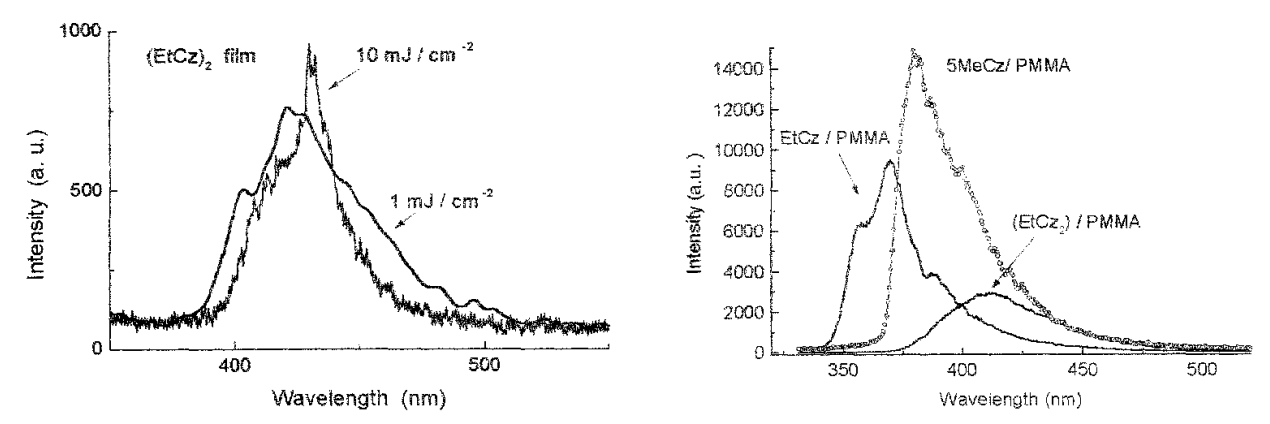

Figure 1. Spectres de tworescence de composés carbazoliques excités â $313 \mathrm{~nm}$

L'excitation laser à $313 \mathrm{~mm}$ de ces différents composés carbazoliques produit une luminescence bleue intense autour de $400 \mathrm{~mm}$ (fig.1). En particulier, le pentaméthylcarbazole ( $5 \mathrm{MeCz}$ ), qui peut également être élaboré sous forme d'un polymère (poly5MeCz), apparaît comme un émetteur bleu d'excellent rendement.

\section{RESEAUX PHOTOINDUITS A $193 \mathrm{NM}$}

Les nombreux travaux réalisés sur la photogravure par laser UV de matériaux polymères ont largement démontré les avantages d'une irradiation UV (efficacité, résolution et taille des motifs, minimisation des dommages thermiques) [10-12]. Aussi, pour moduler périodiquement la surface de nos polymères luminescents, sur des profondeurs de quelques dizaines de nanomètres, avons-nous utilisé un laser excimère ArF (193 nm) (Neweks PSX-100, 4ns, $16 \mathrm{~mJ} / \mathrm{cm}^{2}$, divergence $\mathrm{HxV}: 3.5 \times 2.0 \mathrm{mrad}$ ). A cette longueur d'onde la plupart des polymeres sont fortement absorbants et leur structuration par photoablation peut être efficace. Par contre, la faible cohérence spatiale et temporelle du laser excimère nécessite une méthode interférométrique adaptée. Aussi, avons-nous rejeté une méthode interférométrique à division d'amplitude de type Mach-Zender comme celle récemment utilisée dans le visible avec des polymères dopés de molécules photo-sensibles de type azobenzène [13].

\section{Laser UV}

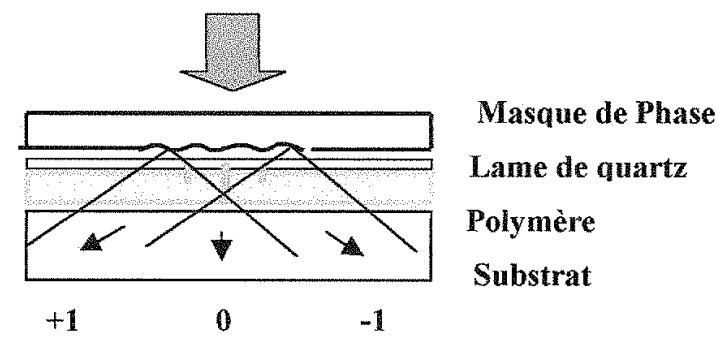

Figmre 2. Irradiation UV dn polymère a travers un masque de phase 
De premiers essais de fabrication de réseaux de pas $(\approx 150 \mathrm{rm})$ ont tout d'abord été réalisés sur des matériaux polymères tels que PMMA, PET ou PVC, en reprenant une technique décrite par S. Lazare et al. [14]. Une structuration périodique submicronique $\Lambda=\lambda(n-\sin \theta)$ de la surface peut être photoinduite (LIPPS) en irradiant le matériau par le laser UV (polarisé à Brewster par une lame de quartz) pour une certaine gamme de densites d'énergie (en dessous du seul d'ablation, $3-7 \mathrm{~mJ} / \mathrm{cm}^{2}$ ) [15]. Mais, cette méthode, très simple, ne permet pas d'obtenir un réseau de qualité sur une longueur de plusieurs millimètres [16]. Il en est de même avec un dispositif interféromètrique à miroir de Lloyd [17-18] qui permet de fabriquer des réseaux de pas $\Lambda$ variable par simple rotation $\theta$ du dispositif miroir/polymère $(\Lambda=$ $\left.\lambda_{\text {pump }} / 2 \sin \theta\right)$ mais sur une zone d'interférence étroite $(2 \mathrm{~mm})$.

L'irradiation du film polymère à travers un masque de phase (fig. 2) s'est révélée la meilleure méthode pour fabriquer une structure DFB [19-21]. Le premier masque de phase utilisé de pas $\Lambda=1090$ $\mathrm{nm}$ nous a permis, à partir de la figure d'interférence entre les ordres $0, \pm 1$, de fabriquer à la surface du matériau un réseau en relief d'excellente qualité et de pas $\Lambda$ identique à celui du masque. Un espacement $\Delta$ entre le masque et le film permet léjection de la matière organique dans le processus de photo-ablation. Une lame de quartz peut être insérée entre le film polymère et le masque pour protéger ce dernier.

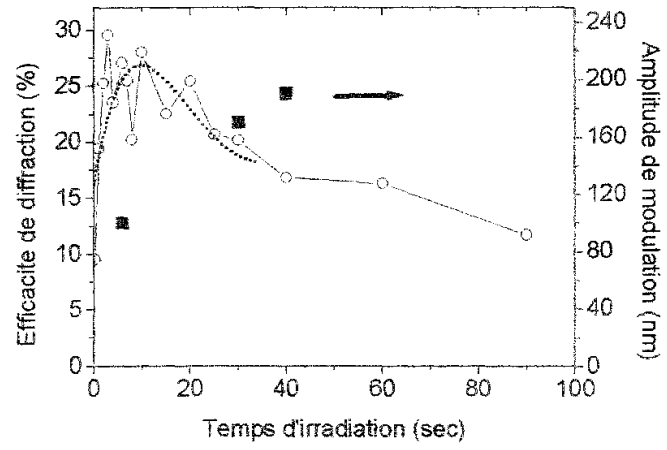

Figure 3. Amplitude des modulations en fonction du temps d'irradiation pour une densité d'energic du laser de $25 \mathrm{~mJ} / \mathrm{cm}^{2}$

La lisibilité des franges d'interférence [19-20], qui dépend de la longueur de cohérence du laser $\operatorname{ArF}(\delta \approx 200 \mu \mathrm{m})$, est encore bonne pour un espacement $\Delta<\delta / 2 \tan \theta(\Delta \approx 1100 \mu \mathrm{m}), \theta$ correspondant à l'angle de diffraction pour les ordres $m= \pm 1$. Les densités d'énergie du faisceau laser $\left(10-60 \mathrm{~mJ} / \mathrm{cm}^{2}\right)$ ainsi que les temps d'irradiation (2-60 sec) sont optimisés en fonction de la nature des polymères.

Dans le cas du PET (seuil d'ablation $17 \mathrm{~mJ} / \mathrm{cm}^{2}$ à $193 \mathrm{~mm}$ ), l'amplitude des modulations étudiée au microscope a force atomique (AFM) montre un effet de saturation en fonction de la dose (fig. 3). La saturation observée $(\approx 200 \mathrm{~nm})$ s'explique par un dépôt progressif des fragments organiques sur le film polymère (fig. 4c). A cause de ce dépot, l'efficacité de diffraction du réseau (fig.3), étudiée avec un laser HeNe en fonction du temps d'itradiation UV, passe par un maximum pour décroitre ensuite du fait de la très forte diffision.
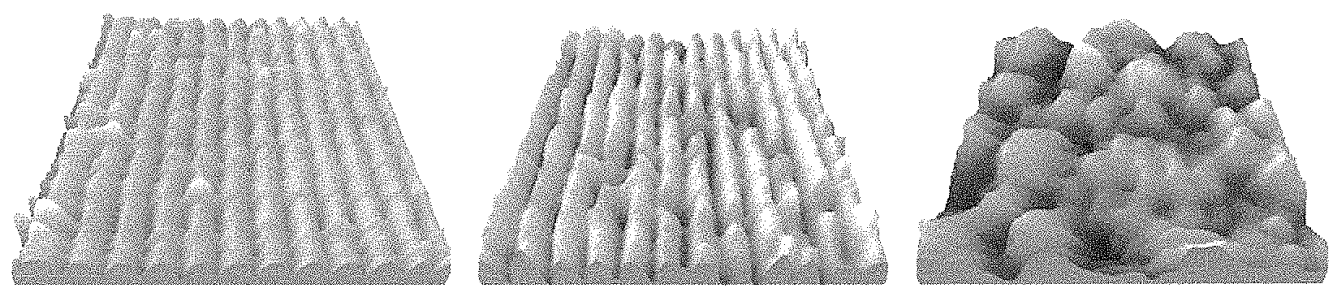

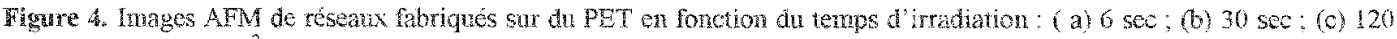
sec pour $F=25 \mathrm{~m} / \mathrm{cm} \mathrm{cm}^{2}$ 
D'excellents réseaux de pas $\Lambda=1090 \mathrm{~nm}$ ont également été fabriqués avec des films de PMMA (épaisseur $700 \mathrm{~nm}$ ) dopés de rhodamine $6 \mathrm{G}$ et déposés sur un substrat de silice. Bien que les conditions de rétroaction $D F B$ ne soient pas favorables (ordre $m=6$ du réseau de Bragg), nous avons pu mettre en évidence, par pompage du film à $532 \mathrm{~nm}$, une émission stimulée monomode à $556.6 \mathrm{~nm}$ en accord avec les conditions de propagation d'un mode $\mathrm{TE}_{0}$ dans une cavité de type planaire et DFB (fïg.5). Mais, cette émission laser est observée pour une densité d'énergie de $1 \mathrm{~J} / \mathrm{cm}^{2}$ alors que dans une étude similaire avec un film de même nature, mais structuré par un réseau de pas $\Lambda=386 \mathrm{~nm}$ et donc travaillant dans "'ordre $\mathrm{m}=2, C$. Fiorini et al obtiennent une émission laser à $567 \mathrm{~nm}$ pour une densité d'énergie de $0.4 \mathrm{mI} / \mathrm{cm}^{2}$ [22]. Ce résultat confirme la nécessité de travailler à l'ordre de Bragg $m=1$ ou 2 et donc de fabriquer sur les polymères émettant autour de $400 \mathrm{~m}$ des réseaux de pas submicronique $\Lambda=m 400 / 2 \mathrm{n}_{\text {eff }}$.

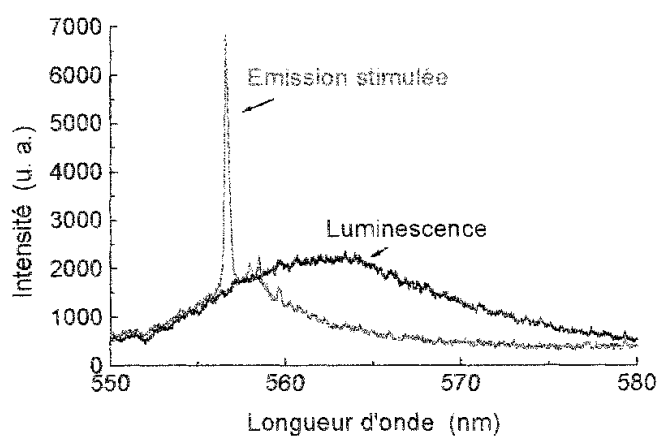

Figure 5. Emission d'un film de PMMA dopé de Rh6G pompé a 532 nm en microcavité DFB planaire $(A=1090$ nm)

Dans ce but nous avons utilisé un masque de phase ibsen de pas $A=250 \mathrm{~nm}$ que nous avons protégé des débris d'ablation par une lame de silice d'épaisseur $80 \mu \mathrm{m}$ placée entre le masque et le film. Ce masque est calculé pour travailler à l'angle de Bragg $\left(\sin \theta_{\operatorname{Bragg}}=\lambda 2 \Lambda\right)$ à $193 \mathrm{~nm}$ créant dans ces conditions des interférences entre les ordres 0 et +1 avec un bon contraste jusqu'à une distance masque/film polymère $\Delta=230 \mu \mathrm{m}$. Un réseau de pas $\Lambda=250 \mathrm{~nm}$ peut être obtenu (fig.6) pour des conditions d'irradiation UV à des densités d'énergie en dessous du seull d'ablation. La modulation du polymère, en contact avec la lame de quartz, s'explique ici par des effets de gradient thermique. Un mouvement de matière se produit des zones fluides (franges brillantes) vers les zones solides (franges sombres). De tels mouvements photo-induits mettent en cause différents mécanismes actuellement en cours d'étude [23].
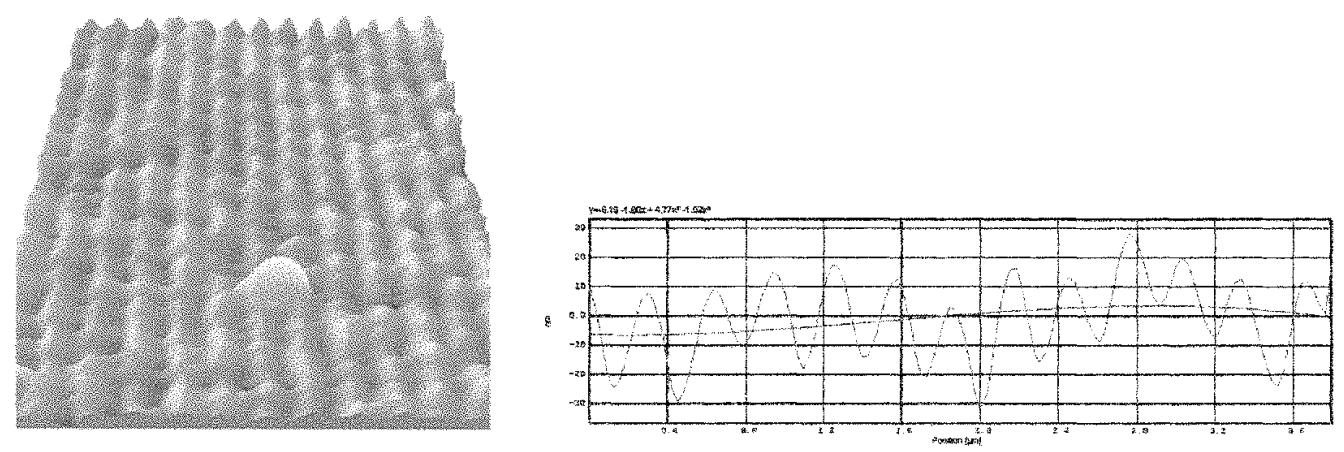

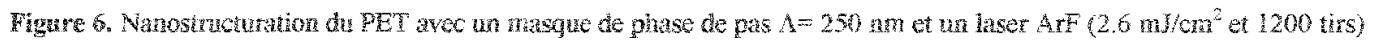




\section{CONCLUSION}

La fabrication de réseaux de pas sub-micronique par irradiation UV (193 nm) d'un masque de phase en contact avec un film polymère est une méthode simple. Il est maintenant possible d'envisager la réalisation d'une micro-cavité DFB planaire pour un laser plastique bleu.

\section{Remerciements}

Nous remercions C. Fiorini, P.A. Chollet et $L$. Rocha du CEA-DRT-LIST pour leur aide apportée dans l'analyse AFM des réseaux.

\section{Références}

[1] Braun D., Materialstoday, 5 (2002) 32-39

[2] Wegmann G., Giessen H., Greiner A, Mahrt R.F., Phys. Rev. B, 57 (1998) R4218-R4221

[3] Schon J.H., Kloc C., Dodabalapur A., Batlogg B., Science 289 (2000) 599-601

[4] Hide F., Diaz-Garcia M.A., Schwartz B.J., Andersson M.R., Pei Q., Heeger A.J., Science, 273 (1996) $1833-1836$

[5] Kraft A., Grimsdale A.C., Holmes A., Angew. Chem. Int. Ed. 37 (1998) 402-428

[6] Kogelnik H., Shank C.V., Appl. Phys. Lett. 18 (1971) 152-154

[7] Kim D.Y., Cho H.N., Kim C. Y., Prog. Polym. Sci. 25 (2000) 1089-1139

[8] Ades D., Boucard V., Cloutet E., Siove A., Olivero C., Castex M.C., Pichler G., J. of Appl. Phys, 87 (2000) $7290-7293$

[9] Cloutet E., Olivero C., Ades D., Castex M.C., Siove A., Polymers, 43 (2002) 3489-3495

[10] Srinivasan R., Braren B., Seeger D.E., Dreyrus R.W., Macromole. 19 (1986) 916-921

[11] Bauerle D., Laser Processing and Chemistry, $3^{\text {rd }}$ ed. (Springer, Heidelberg 2000)

[12] Bityurin N., Malyshey A., J. Appl. Phys. 92 (2002) 605-613

[13] Rocha L., Dumarcher V., Malcor E., Fiorini C ., Denis C., Raimond P., Geffroy B., Nunzi J.M., Synthetic. Met. 127 (2002) 75-79

[14] Bolle M., Lazare S., J. Appl. Phys. 73 (1993) 3516-3524

[15] Csete M., Bor Z., Appl. Surf. Sci. 133 (1998) 5-16

[16] Olivero C.,Castex M.C., Pichler G., Adès D., Cloutet E., Siove A., J. Phys. IV, 11 (2001 61-62

[17] Barrett C.J., Natansobn A.L., Rochon P.L., J. Phys. Chem, 100 (1996) 8836-8842

[18] Kranzelbinder G., Toussaere E., Josse D., Zyss J., Synt. Metals, 121 (2001) 1617-1620

[19] Dyer P.E., Farley R.J., Giedl R., Opt. Comm. 115 (1995) 327-334

[20] Dyer P.E., Farley R.J., Giedl R., Opt. Comm. 129 (1996) 98-108

[21] Klein-Wiele, J.H., Bader M.A., Bauer I., Soria S., Simon P., Marowsky G., Synth. Met. 127 (2002) 53-57

[22] V. Dumarcher, L. Rocha, C. Denis, C. Fiorini, J.M. Nunzi, F. Sobel, B. Salraoui and D. Gindre, J. Opt. A : Pure Appl.Opt 2 (2000) 279

[23] Geue T.M., Saphiannikova MG., Henneberg O., Pietsch U., Rochon P.L., Natansohn A.L., Phys. Rev E, 65 (2002) $52801-52804$ 